\title{
Coherent Showers for the LHC
}

\author{
Nadine Fischer* \\ Monash University \\ E-mail: nadine.fischeremonash.edu \\ Peter Skands \\ Monash University \\ E-mail: peter.skands@monash.edu
}

We present a full-fledged antenna shower for hadron collisions in the VINCIA framework and focus on initial state radiation. The current version of the shower is limited to massless QCD partons and colourless resonances. As a proof-of-concept for the applicability of tree-level matrixelement corrections a la GKS we study $Z$ boson production at the LHC, corrected up to Born + $\mathscr{O}\left(\alpha_{s}^{2}\right)$, and show some first results with matrix-element corrections.

12th International Symposium on Radiative Corrections (Radcor 2015) and LoopFest XIV (Radiative Corrections for the LHC and Future Colliders)

15-19 June 2015

UCLA Department of Physics \& Astronomy Los Angeles, CA, USA

${ }^{*}$ Speaker. 


\section{Introduction}

Parton-shower Monte Carlo programs, which allow to predict the full final-state kinematics, play an essential role in probing the Standard Model and searching for hints of phenomena beyond our current knowledge. As the LHC experiments enter the second long phase of data collection, the experimental precision will increase, as will the demand for precise and fast tools to provide theory predictions.

In this talk, we will describe the most important ingredients of our QCD antenna shower, focusing on initial-initial and initial-final configurations. We will then briefly review the matrix-element correction procedure and give some details for $Z$ production at the LHC. Finally, we present some first, preliminary results.

\section{Antenna Shower}

A QCD antenna [1,2] represents a colour-connected parton pair, undergoing a $2 \rightarrow 3$ branching process. In other shower models, such as DGLAP [3-5] and Catani-Seymour dipole [6,7] based ones, one parton acts as the emitter, while one or more other partons play the role of the recoiler(s). In the antenna picture there is no such distinction; the antenna is treated as a single entity with a single radiation kernel. This kernel consists of antenna functions used to drive the radiation in the shower, which can be calculated due to the factorization of amplitudes in the soft and collinear limits. For our choice of antenna functions see Ref. [8]. Since the factorization of amplitudes holds in the soft limit, colour coherence is an intrinsic property of the antenna functions.

The kinematics map or recoil strategy specifies how the two on-shell parent parton momenta are replaced with the three on-shell daughter-parton momenta while conserving energy and momentum. The $(n+1)$-particle phase-space factorizes into the $n$-particle and antenna phase-space, $\mathrm{d} \Phi_{n+1}=\mathrm{d} \Phi_{\text {ant }} \mathrm{d} \Phi_{n}$, depending on the specific choice of the kinematics map. The antenna phasespace constitutes an important ingredient in the construction of the shower.

Notation The partons will be labelled as follows: capital letters for pre-branching (parent) and lower-case letters for post-branching (daughter) partons, as in $I K \rightarrow i j k$. The first letters of the alphabet, $a, b$, are used for incoming partons, while outgoing ones are denoted by $i, j, k$. We denote the recoiler (more generally the recoiling system) by $R$ and $r$ respectively.

We restrict ourselves to massless partons and denote the branching invariant between two partons $x$ and $y$ by $s_{x y} \equiv 2 p_{x} p_{y}=\left(p_{x}+p_{y}\right)^{2}$, which is always positive regardless of whether the partons involved are in the initial or final state.

We will denote the evolution variable by $t$; it is evaluated on the post-branching branching partons, hence, e.g., $t_{\mathrm{IF}}=t\left(s_{a j}, s_{j k}\right)$.

No-emission Probability A shower algorithm is based on the probability that no branching occurs between two scales $t_{\text {start }}$ and $t_{\text {end }}$, with $t_{\text {start }}>t_{\text {end }}$. In the case of an initial-initial antenna $A B$ with emission $j$, the no-emission probability reads

$$
\Pi\left(t_{\text {start }}, t_{\text {end }}\right)=\exp \left(-\int_{t_{\text {end }}}^{t_{\text {start }}} \mathrm{d} \Phi_{\text {ant }} 4 \pi \alpha_{s} \mathscr{C} \frac{f_{a}\left(x_{a}, t\right)}{f_{A}\left(x_{A}, t\right)} \frac{f_{b}\left(x_{b}, t\right)}{f_{B}\left(x_{B}, t\right)} \bar{a}_{A B j}\left(s_{a j}, s_{j b}, s_{A B}\right)\right),
$$



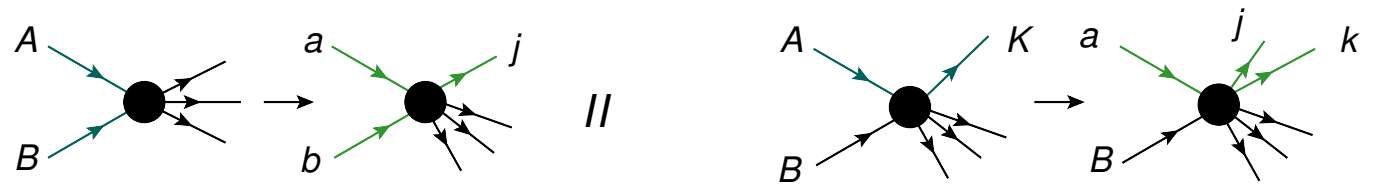

IF

Figure 1: Illustration of initial-initial, $A B \rightarrow a b j$, and initial-final branchings, $A K \rightarrow a k j$. For the II case, the recoil absorbed by the hard system is illustrated by the change in orientation of the three outgoing lines representing the original final-state system.

with the strong coupling $\alpha_{s}$, colour factor $\mathscr{C}$, colour- and coupling-stripped antenna function $\bar{a}_{A B j}$ and the (double) ratio of PDFs,

$$
R_{\mathrm{pdf}}=\frac{f_{a}\left(x_{a}, t\right)}{f_{A}\left(x_{A}, t\right)} \frac{f_{b}\left(x_{b}, t\right)}{f_{B}\left(x_{B}, t\right)} .
$$

Initial-Initial Configurations An initial-initial branching is denoted by $A B \rightarrow a b j$ with the recoiling system $R \rightarrow r$, which consists of all partons produced in the collision $A+B \rightarrow R$, see the left side of fig. 1. We define our kinematics maps to keep the direction of the beam fixed and to preserve the invariant mass as well as the rapidity of the recoiling system: $m_{r}^{2}=m_{R}^{2}$ and $y_{r}=y_{R}$.

The antenna phase-space expressed in the branching invariants reads

$$
\mathrm{d} \Phi_{\text {ant }}^{\mathrm{II}}=\frac{1}{16 \pi^{2}} \frac{s_{A B}}{s_{a b}^{2}} \mathrm{~d} s_{a j} \mathrm{~d} s_{j b} \frac{\mathrm{d} \phi}{2 \pi} .
$$

The evolution variable for gluon emission is the physical transverse momentum of the emission,

$$
t_{\mathrm{II}}^{\mathrm{emit}}=p_{\perp \mathrm{II}}^{2}=\frac{s_{a j} s_{j b}}{s_{a b}}=\frac{s_{a j} s_{j b}}{s_{A B}+s_{a j}+s_{j b}},
$$

which exhibits the same symmetry as the leading poles of the corresponding antenna functions. Constant contours of $p_{\perp \mathrm{II}}^{2}$ are shown in fig. $2 a$ ), as a function of the two branching invariants $s_{a j}$ and $s_{j b}$. The upper phase-space bound is $s_{A B}+s_{a j}+s_{j b} \leq s$, where $s$ denotes the hadronic centre-of-mass energy squared, and defines the hypotenuse of the triangular shape.

For branchings with flavour changes in the initial state the antenna functions contain only single poles. We then use the corresponding invariant, $s_{a j}$ or $s_{j b}$ respectively, as evolution variable,

$$
t_{\mathrm{II}}^{\text {conv }}=Q_{\mathrm{II}}^{2}=\left\{\begin{array}{l}
s_{a j} \text { for } a \text { converting to/from a gluon } \\
s_{j b} \text { for } b \text { converting to/from a gluon }
\end{array} .\right.
$$

The complementary phase-space variable $\zeta$, which has to be linearly independent of $t$ with only one possible mapping between $\left(s_{a j}, s_{j b}\right)$ and $(t, \zeta)$, is chosen to make the integrands and phase-space boundaries in the no-emission probability as simple and efficient as possible. An example for gluon emission is $\zeta=s_{a j} / s_{a b}$. 

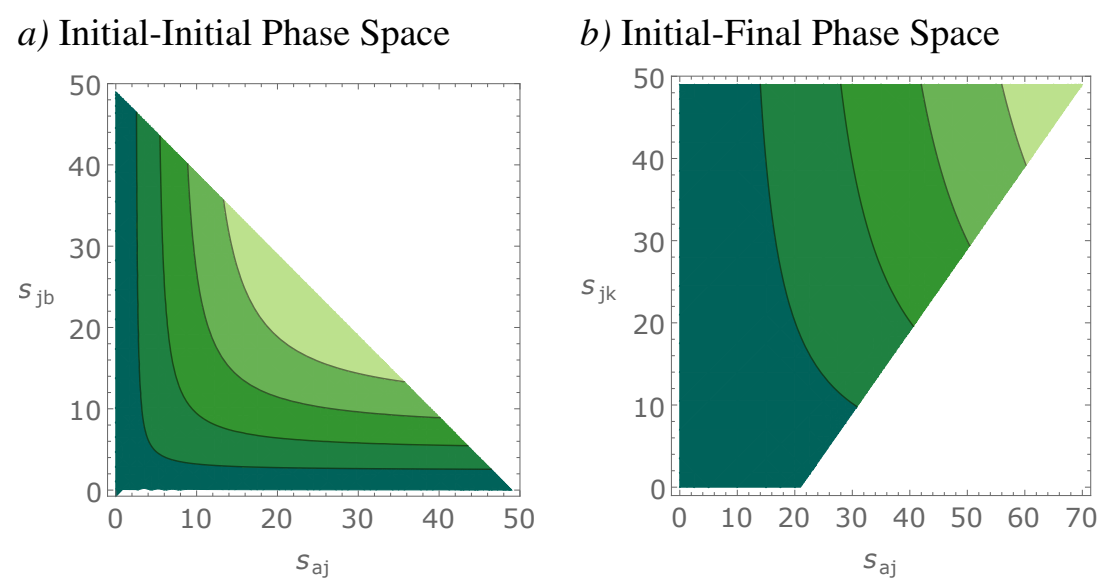

Figure 2: Contours of constant gluon-emission evolution variable for $a$ ) initial-initial and $b$ ) initialfinal configurations. For $a$ ) the recoiler is chosen to be a Higgs boson, $s_{A B}=m_{H}^{2}$, and for $b$ ) $s_{A K}=10500 \mathrm{GeV}^{2}$ and $x_{A}=0.3$. For both cases, the total hadronic $\sqrt{s}=7 \mathrm{TeV}$.

Initial-Final Configurations We denote the pre- and post-branching partons participating in an initial-final branching by $A K \rightarrow a k j$. We define our kinematics map to keep the direction of the beam fixed and to conserve momentum locally within the antenna, leaving the momentum of the spectators unchanged, $p_{b}=p_{B}$ and $p_{r}=p_{R}$, see the right-hand side of fig. 1 .

The antenna phase-space expressed in the branching invariants reads

$$
\mathrm{d} \Phi_{\mathrm{ant}}^{\mathrm{IF}}=\frac{1}{16 \pi^{2}} \frac{s_{A K}}{\left(s_{A K}+s_{j k}\right)^{2}} \mathrm{~d} s_{a j} \mathrm{~d} s_{j k} \frac{\mathrm{d} \phi}{2 \pi} .
$$

We evolve gluon emission in the transverse momentum of the emission, defined as

$$
t_{\mathrm{IF}}^{\mathrm{emit}}=p_{\perp \mathrm{IF}}^{2}=\frac{s_{a j} s_{j k}}{s_{A K}+s_{j k}}=\frac{s_{a j} s_{j k}}{s_{a k}+s_{a j}} .
$$

Constant contours of $p_{\perp \text { IF }}^{2}$ are shown in fig. $2 b$ ), as a function of the two branching invariants $s_{a j}$ and $s_{j k}$. Note that the phase-space is limited by $s_{j k} \leq s_{A K}\left(1-x_{A}\right) / x_{A}$ and $s_{a j} \leq s_{A K}+s_{j k}$.

For branchings with flavour changes in the initial or final state we use the corresponding invariant, $s_{a j}$ or $s_{j k}$ respectively,

$$
t_{\mathrm{IF}}^{\text {conv }}=Q_{\mathrm{IF}}^{2}=\left\{\begin{array}{l}
s_{a j} \text { for } a \text { converting to/from a gluon } \\
s_{j k} \text { for } K \rightarrow q \bar{q}
\end{array} .\right.
$$

As for initial-initial configurations, the complementary phase-space variable $\zeta$ is chosen to simplify the no-emission probability. An example for gluon emission is $\zeta=s_{A K} /\left(s_{A K}+s_{j k}\right)$.

\section{Matrix-Element Corrections for $p p \rightarrow Z+X$}

We review the GKS procedure for iterative matrix-element corrections (MECs), which has successfully been used to include MECs through $\mathscr{O}\left(\alpha_{s}^{4}\right)$ for hadronic $Z$ decays in [9]. Here, we apply it to initial state radiation in $p p \rightarrow Z$ up to $\mathscr{O}\left(\alpha_{s}^{2}\right)$. 
MECs take the all-orders approximation of the shower as their starting point, and apply finite multiplicative correction factors to this structure order by order in perturbation theory. This correction factor essentially replaces the antenna function by the corresponding leading order (LO) matrix element,

$$
\mathscr{O}\left(\hat{t}_{i}, t_{i}\right) \mathscr{C}_{i} \bar{a}_{i} \rightarrow \mathscr{O}\left(\hat{t}_{i}, t_{i}\right) \mathscr{C}_{i} \bar{a}_{i} P_{n}^{\mathrm{ME}} \quad \text { with } \quad P_{n}^{\mathrm{ME}}=\frac{\left|\mathscr{M}_{n}\right|^{2}}{\sum_{j} \mathscr{O}\left(\hat{t}_{j}, t_{j}\right) \mathscr{C}_{j} \bar{a}_{j}\left|\mathscr{M}_{n-1}\right|^{2}},
$$

with the $n$-parton matrix-element correction factor $P_{n}^{\mathrm{ME}}$, scale of the current branching $t_{i}$ and the function $\mathscr{O}\left(\hat{t}_{i}, t_{i}\right)$ expressing the ordering of the shower, with respect to a reference scale $\hat{t}_{i}$. The sum in the denominator of the correction factor runs over all possible ways the shower could have produced the $n$ parton out of the $n-1$ parton state. Traditional showers have dead zones, as they are strongly ordered with $\mathscr{O}\left(\hat{t}_{i}, t_{i}\right)=\mathscr{O}\left(t_{i-1}, t_{i}\right)=\Theta\left(t_{i-1}-t_{i}\right)$, where $t_{i-1}$, the scale of the last branching, acts as reference scale. This requires the combination of different event samples to fill all of phasespace. To avoid dead zones to begin with, we allow our shower to start at the phase-space maximum for the first branching and, starting from the second branching, to produce unordered branchings, suppressed with the factor

$$
\mathscr{O}\left(\hat{t}_{i}, t_{i}\right)=\frac{\hat{t}_{i}}{\hat{t}_{i}+t_{i}},
$$

where the scale $\hat{t}_{i}$ is calculated based solely on the $n-1$ parton state at hand. $\hat{t}_{i}$ is the smallest of all scales, associated with the branchings from any $(n-2)$ to the $(n-1)$-parton state. All antennae involved in the branching whose scale entered in the determination of $\hat{t}_{i}$ are allowed to restart their evolution at the phase-space limit, whereas all other antennae use $\hat{t}_{i}$ as restart scale. This Markovian setup ensures that in the denominator of the MEC factor in eq. (3.1) the clustering has to performed only one step back, and not all the way to the corresponding Born phase-space point. Note that, however, the factorization scale used to evaluate the PDFs is never allowed to become larger during the shower evolution.

Shower Starting Scale and PDFs Note that the following paragraph represents a snapshot of what was done during the development of the shower at the time the talk was given. The method described here is now not our preferred choice, which will be explored in Ref. [10]. As mentioned before, to apply the MECs in $Z$ production, we start the shower from the phase-space maximum, the hadronic centre-of-mass energy squared $s$. To do so we use the same scale as factorization scale in the hard process and apply a reweighting procedure, resulting in the following Born exclusive cross section at the shower cutoff scale $\mu$,

$$
\Pi(s, \mu) f_{0}\left(x_{0}, m_{Z}^{2}\right)\left|\mathscr{M}_{Z}\right|^{2} \mathrm{~d} \Phi_{Z} .
$$

For all higher orders we choose the reweighting such that the PDFs appear with a factorization scale that is the maximum of the $Z$ mass squared and the scale of the first branching, e.g., for the Born +1 exclusive cross section with the matrix element correction factor already applied,

$$
\Pi\left(t_{2}^{\max }, \mu\right) \frac{f_{1}\left(x_{1}, t_{1}\right)}{f_{0}\left(x_{0}, t_{1}\right)} \Pi\left(s, t_{1}\right) f_{0}\left(x_{0}, \max \left(m_{Z}^{2}, t_{1}\right)\right)\left|\mathscr{M}_{Z+1}\right|^{2} \mathrm{~d} \Phi_{Z+1},
$$

where $t_{2}^{\max }$ is the phase-space maximum for the second branching, as the shower is allowed to produced unordered branchings. 
An Example We consider the matrix-element correction to $Z$ production with gluon emission only. We use RАмво [11] to generate large samples of uniformly distributed $p p \rightarrow Z g g$ phasespace points and cluster them back to the corresponding $p p \rightarrow Z$ phase-space point, using the exact inverse of the $2 \rightarrow 3$ recoil prescription of the shower as a clustering algorithm. To compare the shower approximation with the LO matrix element for $q_{1} \bar{q}_{2} \rightarrow Z g_{3} g_{4}$, we use the tree-level PS-toME ratio (the inverse of the matrix-element correction factor above)

$$
\begin{aligned}
R_{3}= & \frac{\mathscr{O}\left(t_{\widehat{43}}, t_{3}\right) \mathscr{C}_{q g} \bar{a}_{q g g}^{\mathrm{IF}}(1,4,3) \mathscr{C}_{q \bar{q}} \bar{a}_{q \bar{q} g}^{\mathrm{II}}(\widehat{13}, 2, \widehat{43})\left|\mathscr{M}_{Z}(Z)\right|^{2}}{\left|\mathscr{M}_{Z g g}(1,2 ; Z, 3,4)\right|^{2}} \\
& +\frac{\mathscr{O}\left(t_{\widehat{34}}, t_{4}\right) \mathscr{C}_{\bar{q} g} \bar{a}_{\bar{q} g g}^{\mathrm{IF}}(2,3,4) \mathscr{C}_{q \bar{q}} \bar{a}_{q \bar{q} g}^{\mathrm{II}}(1, \widehat{24}, \widehat{34})\left|\mathscr{M}_{Z}(Z)\right|^{2}}{\left|\mathscr{M}_{Z g g}(1,2 ; Z, 3,4)\right|^{2}}
\end{aligned}
$$

where hatted variables denote clustered momenta. The two terms correspond to the two possible shower paths, where either gluon 3 or gluon 4 is clustered first. The corresponding sequential clustering scales are

$$
t_{3}=p_{\perp \mathrm{IF}}^{2}\left(g_{3}\right), t_{\widehat{43}}=p_{\perp \mathrm{II}}^{2}\left(g_{\widehat{43}}\right) \quad \text { and } \quad t_{4}=p_{\perp \mathrm{IF}}^{2}\left(g_{4}\right), \quad t_{\widehat{34}}=p_{\perp \mathrm{II}}^{2}\left(g_{\widehat{34}}\right) .
$$

In fig. 3 we show the average of the tree-level PS-to-ME ratio $\left\langle R_{3}\right\rangle$ for $p p \rightarrow Z g g$, differentially over the 4-parton phase-space. The $x$ axis characterizes the scale of the first emission, $p_{\perp \mathrm{II}}^{2}$, normalized to the $Z$ mass, while the $y$ axis is the scale of the second emission, $p_{\perp \text { IF }}^{2}$, normalized to the scale of the first emission, $p_{\perp \text { II }}^{2}$. Note that from the two possible shower paths, the more singular one, the one where the scale of the second emission is smaller, is used to characterize the phase-space point. The left plot in fig. 3 shows the shower with strong ordering conditions,

$$
\mathscr{O}\left(t_{\widehat{43}}, t_{3}\right)=\Theta\left(t_{\widehat{43}}-t_{3}\right) \quad \text { and } \quad \mathscr{O}\left(t_{\widehat{34}}, t_{4}\right)=\Theta\left(t_{\widehat{34}}-t_{4}\right)
$$

The strongly ordered shower produces a dead zone as there are no phase-space points contributing to $y$-values larger than zero, since those correspond to scales $p_{\perp \mathrm{IF}}^{2}>p_{\perp \mathrm{II}}^{2}$. In the strongly ordered region defined by $p_{\perp \text { IF }}^{2} \ll p_{\perp \text { II }}^{2} \ll m_{Z}^{2}$ (the black box in the plots), the shower describes the treelevel matrix element very well, as expected. The right plot in fig. 3 shows the shower with smooth ordering conditions,

$$
\mathscr{O}\left(t_{\widehat{43}}, t_{3}\right)=\frac{t_{\widehat{43}}}{t_{\widehat{43}}+t_{3}} \quad \text { and } \quad \mathscr{O}\left(t_{\widehat{34}}, t_{4}\right)=\frac{t_{\widehat{34}}}{t_{\widehat{34}}+t_{4}}
$$

These conditions remove the dead zone, while not changing the shower in the strongly ordered region. The shower is quite well behaved in the region $p_{\perp \mathrm{IF}}^{2}>p_{\perp \mathrm{II}}^{2}$, but shows some larger deviations to the tree-level matrix element at the edge of phase-space in the upper right part of the plot. This part of phase-space corresponds to events where the $Z$ boson has been produced as an emission off the initial quarks rather than in the hard process. Thus, a QCD shower will undercount the radiation in this region, as it does not include the corresponding singularities.

\section{Preliminary Results}

As some preliminary results, we show the transverse momentum of the $Z$ boson in $p p \rightarrow$ $Z+X \rightarrow e^{+} e^{-}+X$ events in fig. $4 a$ ) and the cross section for $p p \rightarrow Z+$ jets events in fig. $4 b$ ). 

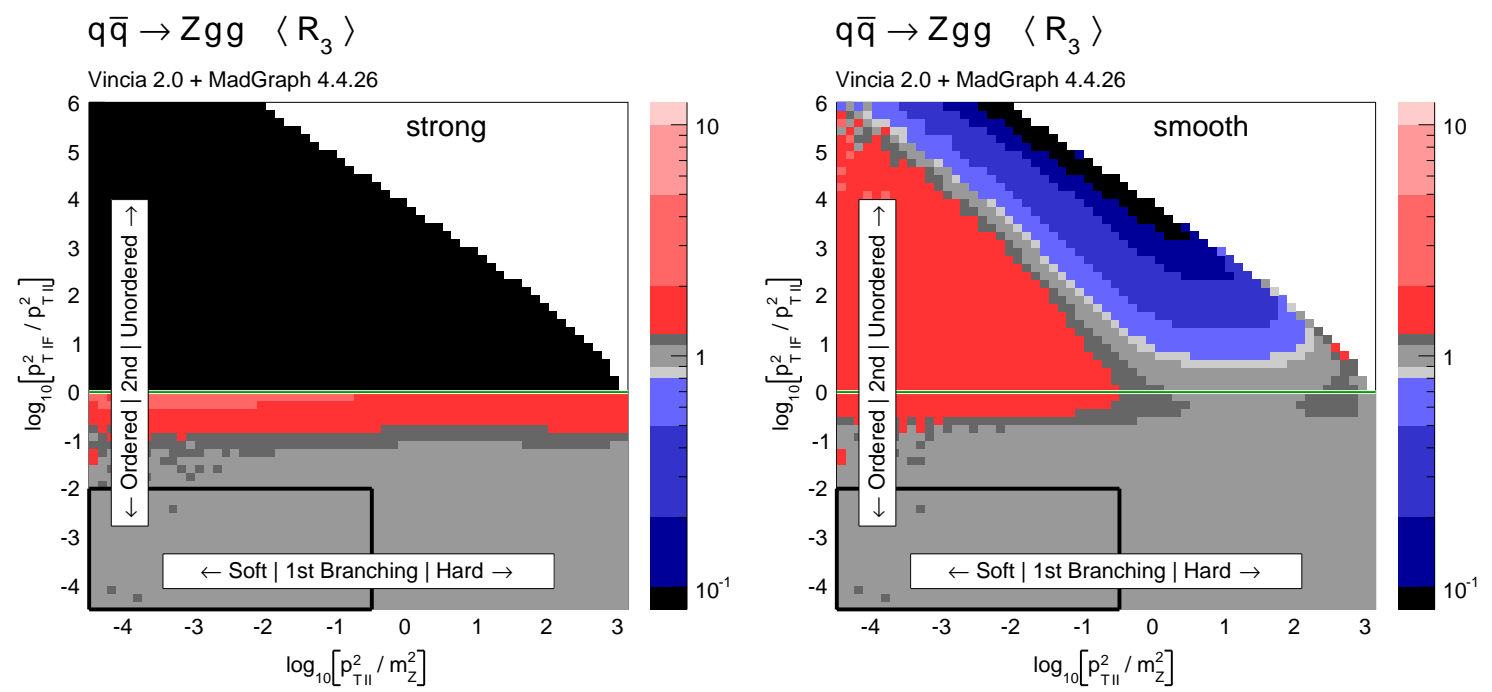

Figure 3: The value of $\left\langle R_{3}\right\rangle$ for $p p \rightarrow Z g g$, differentially over the 4-parton phase-space, with $p_{\perp}^{2}$ ratios characterizing the first and second emissions on the $x$ - and $y$ axis, respectively. Strong (left) and smooth (right) ordering in the shower, with gluon emission only. Leading colour, no sum over colour permutations. Matrix elements generated with MADGRAPH 4 [12].

a) $Z p_{\perp}$ reconstructed from dressed electrons

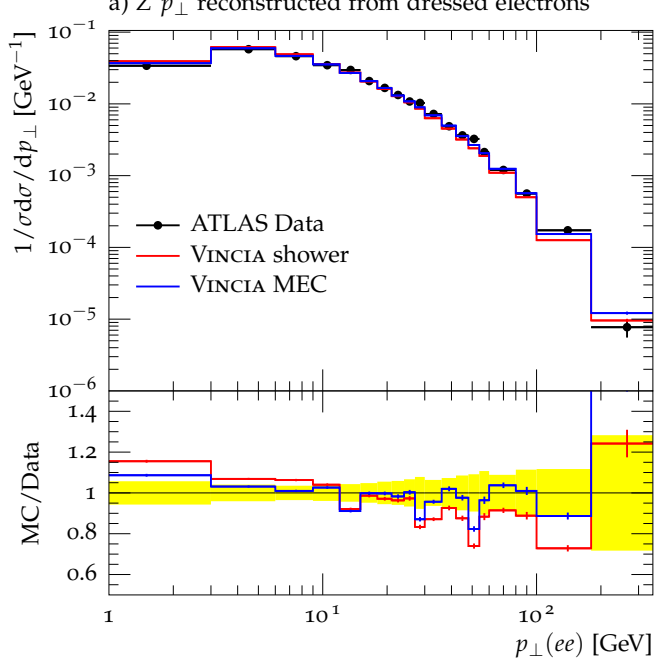

b) $\sigma\left(\geq N_{\text {jet }}\right), Z \rightarrow \ell^{+} \ell^{-}, p_{\perp}$ (jet $)>30 \mathrm{GeV},\left|y_{\text {jet }}\right|<4.4$

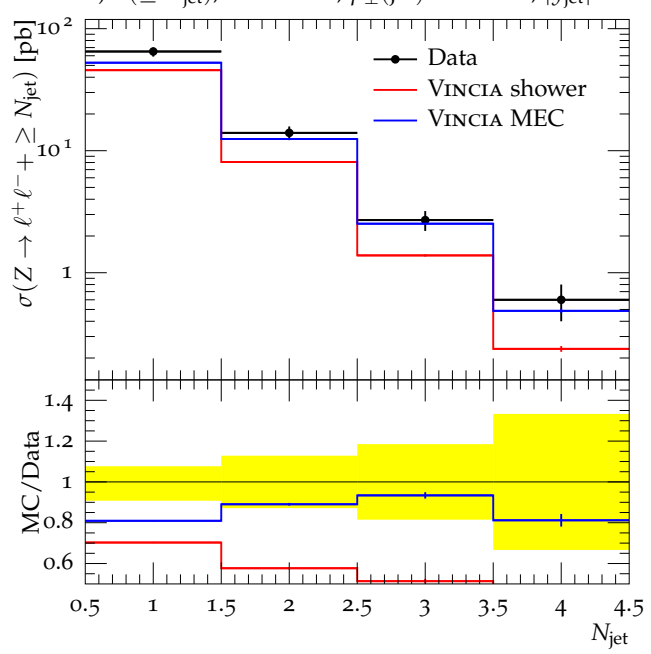

Figure 4: a) $Z$ transverse momentum in $p p \rightarrow Z+X \rightarrow e^{+} e^{-}+X$ events and $b$ ) cross section for $p p \rightarrow Z+$ jets events. Pure shower in red and with MECs in blue. Analysis from RIVET [14].

We use events generated by PYTHIA 8 [13] and shower them with Vincia. In the shower we use a one-loop running of $\alpha_{s}$ with a rather larger reference value of $\alpha_{s}\left(m_{Z}\right)=0.138$. For this value we find a fairly good agreement between the pure shower (red curve) and the data for the $Z$ transverse momentum. By switching MECs on, we find an improved description. To get a reasonable result for the cross section involving additional jets, we need to include the higher-order tree-level matrix elements, as indicated by fig. 4 b). 


\section{Summary and Outlook}

We presented a full-fledged QCD antenna shower for initial state radiation. To improve the description of the shower, we use the iterated matrix-element correction procedure with the example process $p p \rightarrow Z$, corrected up to Born $+\mathscr{O}\left(\alpha_{s}^{2}\right)$.

The development of a more highly automated interface to MADGRAPH 5 [15], as well as the handling of mass effects and resonance decays, are among the main future development targets.

\section{Acknowledgments}

PS is supported in part by the Australian Research Council, contract FT130100744 "Virtual Colliders: high-accuracy models for high energy physics".

\section{References}

[1] G. Gustafson and U. Pettersson, Nucl. Phys. B 306 (1988) 746.

[2] D. A. Kosower, Phys. Rev. D 71 (2005) 045016, [hep-ph/ 0311272 ].

[3] V. N. Gribov and L. N. Lipatov, Sov. J. Nucl. Phys. 15 (1972) 438.

[4] Y. L. Dokshitzer, Sov. Phys. JETP 46 (1977) 641.

[5] G. Altarelli and G. Parisi, Nucl. Phys. B 126 (1977) 298.

[6] S. Catani and M. H. Seymour, Phys. Lett. B 378 (1996) 287, [hep-ph/ 9602277$].$

[7] S. Catani and M. H. Seymour, Nucl. Phys. B 485 (1997) 291, [hep-ph/ 960532 3]. [Erratum: Nucl. Phys. B 510 (1998) 503].

[8] M. Ritzmann, D. A. Kosower, and P. Skands, Phys. Lett. B 718 (2013) 1345, [arXiv: 1210.6345$].$

[9] W. T. Giele, D. A. Kosower, and P. Z. Skands, Phys. Rev. D 84 (2011) 054003, [arXiv:1102.2126].

[10] N. Fischer, S. Prestel, M. Ritzmann and P. Skands, In preparation..

[11] R. Kleiss, W. J. Stirling and S. D. Ellis, Comput. Phys. Commun. 40 (1986) 359.

[12] J. Alwall, P. Demin, S. de Visscher, R. Frederix, M. Herquet, F. Maltoni, T. Plehn, D. L. Rainwater, and T. Stelzer, JHEP 09 (2007) 028, [arXiv: 0706.2334 ].

[13] T. Sjöstrand, S. Ask, J. R. Christiansen, R. Corke, N. Desai, P. Ilten, S. Mrenna, S. Prestel, C. O. Rasmussen, and P. Z. Skands, Comput. Phys. Commun. 191 (2015) 159, [arXiv: 1410.3012 ].

[14] A. Buckley, J. Butterworth, L. Lönnblad, D. Grellscheid, H. Hoeth, J. Monk, H. Schulz and F. Siegert, Comput. Phys. Commun. 184 (2013) 2803, [arXiv:1003.0694].

[15] J. Alwall, R. Frederix, S. Frixione, V. Hirschi, F. Maltoni, O. Mattelaer, H. Shao, T. Stelzer, P. Torrielli and M. Zaro, JHEP 07 (2014) 079, [arXiv: 1405.0301 ]. 